\title{
Tracking the Cognitive Pharmacodynamics of Psychoactive Substances with Combinations of Behavioral and Neurophysiological Measures
}

\author{
Alan Gevins, D.Sc., Michael E. Smith, Ph.D., and Linda K. McEvoy, Ph.D.
}

Many common pharmacological treatments have effects on cognitive ability. Psychometric task batteries used to characterize such effects do not provide direct information about treatment-related changes in brain function. Since overt task performance reflects motivation and effort as well as ability, behavioral measures alone may overestimate or underestimate the impact of a pharmacological intervention on brain function. Here we present a method that combines behavioral and neurophysiological measures in an attempt to detect the psychoactive effects of pharmacological treatments with greater sensitivity than that provided by behavioral measures alone. Initial application of the method is made to the data from a double blind, placebo-controlled, crossover study in which caffeine, diphenhydramine, and alcohol were used to alter the mental state of 16 healthy subjects at rest and while they performed low load and high load versions of a working memory task. For each intervention, more sensitive detection of drug or alcohol effects over a four hour period was obtained when EEG variables were included in multivariate analyses than when only behavioral variables were used. These initial results suggest that it can be useful to incorporate neurophysiological measures of brain activity into inferences concerning the acute impact of drugs on mental function, and demonstrate the feasibility of using multivariate combinations of behavioral and neurophysiological measures to sensitively characterize the pharmacodynamics of drug-induced changes in cognition.

[Neuropsychopharmacology 26:27-39, 2002]

(c) 2001 American College of Neuropsychopharmacology. Published by Elsevier Science Inc. All rights reserved.
KEY WORDS: Pharmacodynamics; Cognition; Attention; Working memory; EEG; Event-related potentials; Multivariate pattern recognition; Diphenhydramine; Caffeine; Alcohol

Many medications affect performance, attention, and alertness. The most common such side effect is sedation (Ramaekers 1998). Patients complain of somnolence, drowsiness, inability to concentrate, and diminished

From the San Francisco Brain Research Institute and SAM Technology, San Francisco, CA

Address correspondence to: Alan Gevins, SFBRI, 425 Bush Street, 5th Floor, San Francisco, CA 94108; Tel.: (415) 837-1699 ext. 133; Fax: (415) 274-9574; e-mail: alan@eeg.com

Received October 30, 2000; revised May 2, 2001; accepted May 25, 2001.

Online publication: 5/30/01 at www.acnp.org/citations/Npp 053001125 . energy. On testing they tend to demonstrate diminished speed and accuracy of psychomotor and cognitive performance (Ramaekers 1998). Psychoactive medications may also impair memory, attention, and concentration in the absence of sedative effects. There is a growing literature on the cognitive side effects of treatments for many types of disorders. For example, recent articles have described acute cognitive impairments associated with interferon- $\alpha$ treatment (Valentine et al. 1998), chemotherapy (van Dam et al. 1998), antianxiety treatments (Sumner 1998), and treatment for allergies (Fireman 1997).

A major problem in determining whether and to what extent drugs produce cognitive effects is that there are no standard effective means for objectively assessing cognitive impairments associated with pharmacological treatments. This lack of a clinical standard has 
been cited as a major confounding factor in the discrepancies between the results of different clinical trials (Vermeulen and Aldenkamp 1995). In most cases performance on an ad hoc battery of rating scales and behavioral tests of cognitive and psychomotor functions is employed. Such tests likely vary widely in their sensitivity. A subtler problem with this approach is the fact that behavior is the end product of many neural systems, some of which may be recruited or adapted in some way to compensate for deficits. That is, an individual might be able to temporarily mobilize the necessary mental resources to perform a cognitive test even when mildly debilitated but not be able to maintain such extra effort over the course of a workday. Conversely, a low level of test performance may reflect motivational rather than ability factors. Hence, in isolation, behavior may not provide an accurate picture of the effects of a medication on cognitive brain function.

Electroencephalogram (EEG) data can provide assessments of cognitive changes that complement the information provided by self-report and behavioral measures. When other factors are held constant, EEG signals tend to have high test-retest reliability (McEvoy et al., 2000; Salinsky et al. 1991). Despite this stability under normal conditions, EEG signals can be very sensitive to variations in alertness (Broughton 1982; Gevins et al. 1977; Makeig and Jung 1995; Matousek and Petersen 1983; Oken and Salinsky 1992; Torsvall and Åkerstedt 1988), and/or the amount of effortful attention exerted during task performance (Gale et al. 1978; Galin et al. 1978; Gevins et al. 1997; Inouye et al. 1988; Miyata et al. 1990). Because of such characteristics, EEG measures have often been used to help characterize the central effects of alcohol (Cohen et al. 1993; Davis et al. 1941; Lukas et al. 1986), and psychoactive medications (Bruce et al. 1986; Hermann 1982; Saletu et al. 1994; Schulz et al. 1996; Semlitsch et al. 1995).

In the context of such research a large number of studies have employed multivariate pattern classification techniques, including both linear discriminant analysis and neural network approaches, in efforts to automatically detect and classify patterns of EEG changes associated with pharmacological interventions. This has included efforts to discriminate the effects of different classes of psychoactive drugs (e.g., stimulants, antidepressants, tranquilizers, and neuroleptics) as an aid in the evaluation of new pharmacological agents (Hermann 1982), to discriminate the effects of different drugs within a class such as different hypnotics used to induce anesthesia (Veselis et al. 1993) and different benzodiazepines used to promote sleep (Gevins et al. 1988), and to examine dose-response relationships (Haring et al. 1994).

Most such pattern classification studies have been conducted using as input data EEG recorded from subjects that were passively resting or even unconscious.,
Recent studies, however, have demonstrated that similar results can be obtained from subjects actively engaged in cognitive task performance. For example, Gevins and Smith (1999) used neural network based methods to compare task-related EEG features between alert and mildly intoxicated states, and between alert and drowsy states, in individual subjects. Using EEG features in the alpha and theta bands, an average crossvalidation classification accuracy of $98 \%$ was obtained across subjects for the alert versus mildly intoxicated comparison (average binomial $p<.0001$ ). Similarly, a cross-validation accuracy of $92 \%$ (range $84 \%-100 \%$ ) was obtained for the alert versus drowsy comparison (average binomial $p<.001$ ). This indicates that task-related EEG variables can be used to detect neurofunctional states associated with mild and transient cognitive impairment.

To our knowledge no studies have yet systematically compared the relatively effectiveness of detecting the psychoactive effects of pharmacological interventions using behavioral versus EEG indices of functional status. Similarly, no reported studies have examined the utility of combining behavioral and neurophysiological measures in multivariate classifiers of drug effects, or have asked whether task related EEG measures complement or are redundant with resting EEG measures. By combining behavioral, resting state EEG measures, and task-related EEG measures it might be possible to detect the effect of medication on CNS function with greater sensitivity. The study reported herein examines this possibility. Multivariate pattern classification methods are applied to behavioral and EEG measures in an attempt to detect the acute CNS effects of several common psychoactive substances (caffeine, alcohol, and the antihistamine diphenhydramine) and to characterize their pharmacodynamics over an extended test session.

\section{METHOD}

\section{Subjects}

All participation was fully informed and voluntary, and the experiment was conducted under appropriate guidelines for the protection of human subjects. Sixteen healthy adults (21-32 years, mean age 26 years, 8 females) received monetary compensation for participation in the study. All subjects were non-smokers, social drinkers (1-10 drinks per week), and moderate consumers of caffeine (1-4 cups of coffee per day). All subjects had consumed antihistamines at some time in the past, but none were currently taking antihistamines or any other psychoactive medications.

\section{Tasks}

Subjects performed two difficulty levels of a continuous performance, $n$-back working memory task (Figure 1), 
versions of which we have employed in many other EEG studies (Gevins and Smith 1999; Gevins and Smith, 2000; Gevins et al. 1996, 1997, 1998; McEvoy et al. 1998; McEvoy et al., 2000; Smith et al. 1999). In this task, subjects were required to compare the spatial location of the current stimulus with that of one presented previously. Briefly, single capital letter stimuli, drawn randomly from a set of twelve, were presented for 200 msec once every $4.5 \mathrm{sec}$ on a computer monitor. At 1.3 sec prior to stimulus onset, a warning cue (a small " $x$ ") appeared in the center of the screen for $200 \mathrm{msec}$. The letter stimulus occurred $1.3 \mathrm{sec}$ after the cue in one of twelve possible locations on the monitor. The identity of the letter and its spatial position varied randomly from trial to trial. A small fixation dot was continuously present at the center of the screen.

In a low load version of the task, subjects were required to match the position of the current stimulus with the position of the very first stimulus presented in the block. In a high load version of the task, subjects compared the current stimulus with that presented two trials previously. In this version subjects were required to remember two positions (and their sequential order) for the duration of two trials (nine seconds), and to update that information on each subsequent trial. In both versions of the task, stimuli were presented in blocks of 53 trials (the first three trials were warm-up trials and were discarded from analysis). Matches occurred randomly on $50 \%$ of the trials. Subjects were instructed to respond as quickly and as accurately as possible.
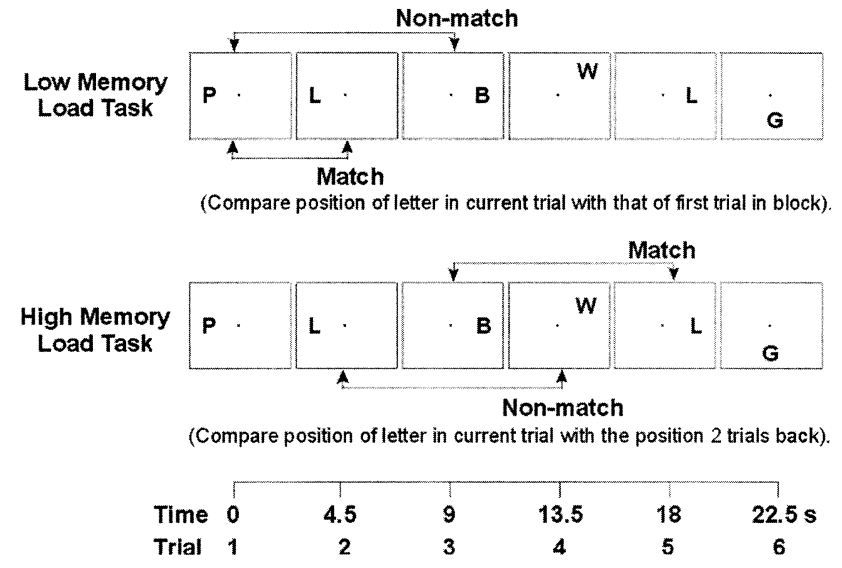

Figure 1. Schematic diagram of the low memory load and high memory load versions of the n-back WM task. Every 4.5 seconds, one of 12 possible capital letter stimuli appeared in one of 12 possible locations on a computer monitor. In the easy version of the task, subjects compared the location of the current stimulus, regardless of its identity as a letter, with the location of the very first stimulus in the block of 50 trials. In the difficult version of the task, subjects compared the location of current stimulus with the location of the stimulus presented two trials previously.

\section{Procedure}

Each subject participated in six sessions. The first session was a practice session in which subjects learned to perform the working memory tasks. Following informed consent procedures, subjects performed 300 trials at each level of the task load. Our prior research has indicated that this is sufficient practice for accuracy and reaction time scores to stabilize in the working memory task. After training, all subjects participated in four sessions, separated by at least one week. These sessions involved recording from subjects after they had ingested alcohol, caffeine, diphenhydramine, or placebo. Eleven of the sixteen subjects returned for an extra session, which was a retest of the diphenhydramine condition.

The four drug sessions were conducted according to a double blind, placebo-controlled, randomized, counterbalanced, crossover design. In each session subjects consumed two pills (unmarked gelatin capsules) and a mixed drink. The pills contained either $50 \mathrm{mg}$ of the antihistamine diphenhydramine (active ingredient in Benadryl), $200 \mathrm{mg}$ of caffeine (equivalent to approximately 2 cups of coffee), or a placebo consisting of powdered sugar. The pills were given with a $500 \mathrm{cc}$ drink containing either $0.88 \mathrm{~g} / \mathrm{kg} 95 \%$ ethanol mixed in fruit juice (adequate to produce an average peak blood alcohol content [BAC] of 0.08), or containing $495 \mathrm{cc}$ of fruit juice with 5 cc of alcohol floated on top to mimic the smell and taste of the treatment drink. The diphenhydramine retest condition was also performed in a double-blind manner, with both the subjects and the experimenters unaware of the nature of the test condition being repeated.

Each drug session involved a baseline recording prior to drug administration. This was followed by four post-drug recording intervals, each lasting approximately 40 minutes. The first interval began $0.5 \mathrm{hr}$ after drug ingestion; the remaining three intervals occurred hourly thereafter. A scientist not otherwise involved in the experiment administered a Breathalyzer test at the beginning of each interval. Subjects also completed the Karolinska (Åkerstedt and Gillberg 1990) and Stanford (Hoddes et al. 1973) sleepiness scales at each test period. Task-related EEG was then recorded while subjects performed two blocks of the low load and high load versions of the working memory task (order of tasks counterbalanced across subjects) and while they rested quietly with their eyes open and closed.

\section{EEG Recording and Preprocessing}

EEG was continuously recorded from 28 scalp electrodes using a digitally linked mastoids reference. EOG was recorded from electrodes placed above and below one eye, and at the other canthi of each eye. Physiological signals were band-pass filtered at 0.01 to $100 \mathrm{~Hz}$ and sampled at $256 \mathrm{~Hz}$. Data were digitally filtered offline 
with a zero phase-shift $0.5 \mathrm{~Hz}$ high pass IIR filter. Automated artifact detection was followed by application of adaptive eye contaminant removal filters (cf. Du et al. 1994). The data were then visually inspected and data segments containing possible residual artifacts were eliminated from subsequent analyses. To obtain power spectra, Fast Fourier transforms were computed on $50 \%$ overlapped, 512 sample Hanning windows for all artifact-free trials and averaged over all data within a condition. Average spectra were converted to $\mathrm{dB}$ power by normalizing them with a $\log _{10}$ transform.

\section{Analyses}

To examine the effects of the pharmacological interventions on individual EEG or behavioral variables, univariate repeated measures analyses of variance (ANOVAs) were used to compare data from each of the three treatment conditions with the placebo condition. For performance measures, accuracy was characterized in terms of the ability to discriminate match trials from non-match trials, or d' (Swets 1964). Reaction times were measured in milliseconds and then normalized prior to statistical analyses. In all cases, the treatment effects were defined as significant Treatment by Recording Interval interactions.

For neurophysiological features, average power was extracted from individual spectral bands at individual electrode sites, and then compared across test conditions. Decisions as to which parameters to extract from the spectra were based on the results of prior studies. For the task related EEG, past studies indicate that the frontal midline theta rhythm and the parietal alpha rhythm are sensitive to variations in the attentional demands of tasks. For example, past research indicates that these parameters are sensitive to the increase in difficulty in the working memory tasks employed here (Gevins and Smith, 2000; Gevins et al. 1997, 1998; McEvoy et al., 2000). In this study, frontal midline theta was measured as the peak frequency between 5 and $7 \mathrm{~Hz}$ at electrode site $\mathrm{AFz}$, and parietal alpha was measured as the average power in a $1 \mathrm{~Hz}$ band around the peak frequency between 8 and $12 \mathrm{~Hz}$ at electrode Pz. For the EEG recorded under passive resting conditions, past studies indicate that activity in the delta and theta bands at posterior sites, and the alpha rhythm measured over the occipital region, are highly sensitive to variations in alertness and arousal (Davis et al. 1937; Gevins et al. 1977; Makeig and Jung 1995; Matousek and Petersen 1983; Oken and Salinsky 1992). Herein, resting condition delta was measured as the average power between 2 and $4 \mathrm{~Hz}$ at $\mathrm{Pz}$, and resting posterior theta was measured as the average power between 4 and $6 \mathrm{~Hz}$ at electrode site Pz. Resting occipital alpha was measured at $\mathrm{Oz}$ as the average power in a $1 \mathrm{~Hz}$ band around the peak frequency in the 8 and $12 \mathrm{~Hz}$ range. Each of the individual features was then compared between placebo and each drug condition in univariate ANOVAs with repeated measures.

In a second series of analyses, multivariate methodsstepwise linear discriminant analysis (LDA) - were used to determine whether the various treatments could be discriminated from placebo. In particular, for each treatment condition (caffeine, alcohol, or diphenhydramine), three sets of stepwise LDAs were performed in which the treatment was compared to the placebo in two-class discrimination problems. The three sets of analyses differed in the constellation of independent variables used. In one, task-related behavioral variables were used (Behavior LDA Analysis). In a second, neurophysiological variables recorded during task performance and during passive resting conditions were used (EEG LDA Analysis). The third used both behavioral and EEG variables (Combined LDA Analysis). In all three analyses, discriminant functions were restricted to a maximum of four variables. In each case LDA functions were derived from a set of measures that included both first-order predictor variables (e.g., performance speed or accuracy, EEG power in particular bands, etc.) and second-order, derived predictor variables. The second-order variables included measures such as changes in variables between high load and tasks, or between eyes-open and eyes closed resting conditions, or ratios of power in different EEG bands.

For each type of analysis, a two-step process was used to analyze the data from each session. First, data from all the post-treatment intervals were submitted to a stepwise LDA to discriminate between treatment and placebo conditions. The variables (features) chosen in this analysis (restricted to a maximum of four) were then submitted together to an LDA to discriminate treatment from placebo data in each interval (including the baseline interval as a control). The LDAs on the baseline interval and on all four post-treatment intervals were performed using a leave-out-one jack-knife cross-validation approach (Efron 1982). In this approach the data from each of the 16 individual subjects was classified using equations that were first derived from the data provided by the other 15 subjects, and then independently applied to the remaining subject. The mean classification accuracy of the 16 such crossvalidation analyses was computed and the significance assessed using the binomial probability distribution. A conservative $p<.01$ criterion was adopted to impute statistical significance to the classification results.

\section{RESULTS}

\section{Subjective Ratings}

Following treatment, participants' self-reports on the Karolinska Sleepiness Scale indicated that they felt 
most alert in the caffeine test condition and least alert during the diphenhydramine test condition (Figure 2). These differences were reflected in a Drug by Recording Interval interaction $(\mathrm{F}(12,168)=6.54 ; p<.001)$. Treatment with caffeine did not produce subjective sleepiness ratings significantly different from placebo. This lack of significant change may reflect a floor effect in the well-rested subjects. Treatment with diphenhydramine led to a significant increase in subjective sleepiness relative to placebo at the $2.5-3 \mathrm{hr}$ and $3.5-4 \mathrm{hr}$ post-treatment intervals. Treatment with alcohol, which reached peak $B A C=0.08$ on average during the first post-treatment interval and which declined steadily to a BAC $=0.03$ on average by the last post-treatment interval, produced a significant increase in subjective sleepiness relative to placebo at the $1.5-2 \mathrm{hr}, 2.5-3 \mathrm{hr}$, and 3.5-4 hr post-treatment intervals. Results with the Stanford Sleepiness Scale were in accordance with these observations.

\section{Summary of Behavioral Performance Effects}

In all drug conditions, subjects responded faster $(\mathrm{F}(1,14)=$ 61.60; $p<.001)$ and more accurately $(\mathrm{F}(1,14)=23.77 ; p<$ .001 ) in the low load WM task condition than in the high load condition. The pattern of drug-related changes in overt task performance was similar to that observed for the subjective ratings (Figure 3), with significant Treatment by Recording Interval interactions for both RT $(\mathrm{F}(12,168)=4.63 ; p<.001)$ and accuracy $(\mathrm{F}(12,168)=4.54 ; p<.001)$. No significant behavioral differences were observed when comparing caffeine to placebo. For the alcohol treatment, no significant main effects were observed for either accuracy or reaction time relative to Placebo. Alcohol did have a more subtle effect on reaction times though, producing a treatment

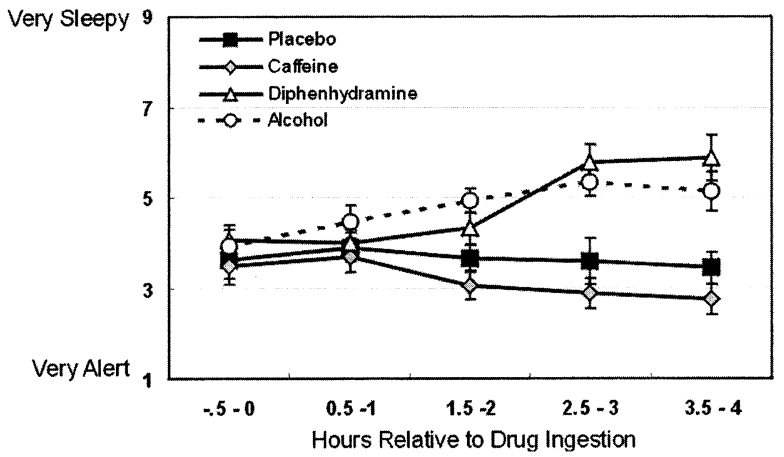

Figure 2. Average $( \pm S E M)$ subjective sleepiness in the placebo, caffeine, diphenhydramine, and alcohol conditions, assessed with the Karolinska Sleepiness Scale. Ratings were obtained during each of five successive intervals beginning pre-treatment $(-.5-0 \mathrm{hr})$ and extending up to four hours post-treatment (3.5-4 hr).
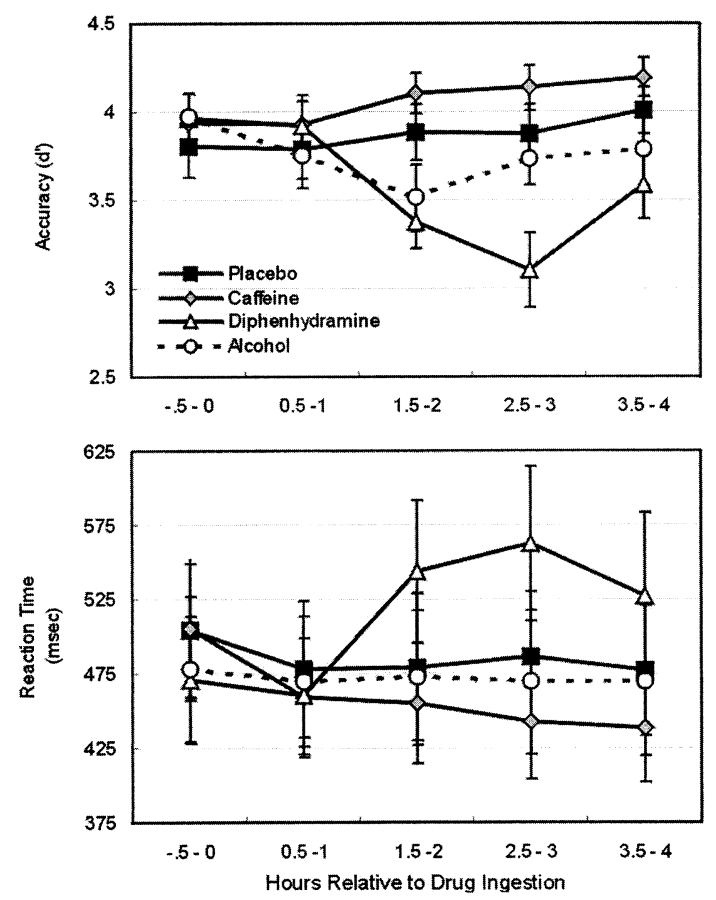

Figure 3. Effects of placebo, caffeine, diphenhydramine and alcohol on average $( \pm S E M)$ accuracy and reaction time of working memory task performance during each of five successive intervals beginning with pre-drug ingestion $(-.5-0 \mathrm{hr})$ and extending up to four hours post-drug ingestion (3.5-4 hr). Accuracy and reaction time measures have been collapsed across difficulty levels. Performance in the diphenhydramine condition was significantly slower and less accurate compared to that in the placebo condition. In contrast, overall performance in the caffeine and alcohol conditions did not greatly differ from performance following the placebo.

by task load interaction whereby reaction times following alcohol were slightly faster in the high load task, and slightly slower in the low load task, relative to responses in the placebo condition $(\mathrm{F}(1,14)=11.60 ; p<$ .01). In contrast, after treatment with diphenhydramine subjects performed the both task levels significantly more slowly $(\mathrm{F}(4,60)=4.98 ; p<.001)$ and less accurately $(\mathrm{F}(4,60)=9.67 ; p<.001)$ than in the placebo condition, with a nadir in the third post-ingestion interval (2.5-3 hours post drug).

\section{Summary of Neurophysiological Effects}

In the placebo condition and in the pretreatment baseline test sessions, neurophysiological parameters varied across the different test conditions in a predictable fashion. For example, alpha band EEG measures were attenuated in the eyes-open resting condition relative to the eyes-closed resting conditions. The major differences in EEG parameters between the high load and low load versions of the WM task also replicated past 
studies (Gevins and Smith, 2000; Gevins et al. 1997, 1998; McEvoy et al., 2000). In particular, the frontal midline theta signal reliably increased with increased task difficulty, and the parietal alpha signal was attenuated in the more difficult task.

When compared with the placebo condition, the different pharmacological treatments resulted in distinct changes to neurophysiological parameters. These changes are summarized in Table 1. Caffeine had the least effect on neurophysiological parameters. Caffeine did not produce significant differences in resting EEG data relative to placebo; however it did produce a significant reduction in alpha band power at parietal sites during the performance of both task conditions $(\mathrm{F}(4,60)=$ $4.91 ; p<$. 01). Alcohol had the largest effect on neurophysiological parameters. Alcohol increased the power in the delta $(\mathrm{F}(4,56)=3.56 ; p<.05)$ and theta $(\mathrm{F}(4,56)=$ $6.73 ; p<.01)$ EEG bands during both resting conditions. During task performance alcohol was associated with an increased in the amplitude of both the frontal midline theta rhythm $(\mathrm{F}(4,56)=12.45 ; p<.01)$ and the parietal alpha rhythm $(\mathrm{F}(4,56)=14.71 ; p<.001)$ in both high load and low load task conditions. Diphenhydramine had large effects on neurophysiological parameters during resting conditions, but relatively subtle effects on the EEG during task performance. In particular, diphenhydramine was associated with an increase in power in the delta $(\mathrm{F}(4,60)=4.37 ; p<.01)$ band during both resting conditions. Power in the theta band also showed a trend towards increasing with diphenhydramine in both resting states $(\mathrm{F}(4,60)=2.83 ; p<.07)$. In contrast, diphenhydramine was associated with an attenuation of power in the alpha band in the eyesclosed resting condition $(\mathrm{F}(4,60)=6.37 ; p<.01)$, but no significant change in power during the eyes-open resting condition. During task performance diphenhydramine was associated with a reduction in power for the frontal midline theta rhythm in the high load task only $(\mathrm{F}(4,60)=3.99 ; p<.05)$. This task-specific reduction in the frontal midline theta rhythm effectively eliminated the difference in frontal midline theta power that was otherwise observed between high load and low load task conditions.

\section{Multivariate Detection of Treatment with Caffeine}

The Behavior LDA Analysis did not produce significant discrimination between the caffeine and placebo conditions in any interval. In contrast, the EEG LDA Analysis significantly discriminated caffeine from placebo beginning with the first post drug interval $(0.5-1 \mathrm{hr}$ post drug; binomial $p<.01$ ). The difference peaked in the second interval (1.5-2 hrs post drug; binomial $p<.001)$, and remained significant throughout the session (Figure 4). This analysis used a combination of resting and task-related EEG features. It consisted of two resting EEG features (posterior delta power recorded during eyes open and eyes closed states) and two second-order task-related EEG features (the difference in frontal midline theta power between the low load and high load task, and the difference in alpha power between the resting, eyes open state and performance of the high load task). At the peak discrimination interval, the highest weights were given to the two resting variables, with slightly greater weight given to the eyes open variable. The frontal midline theta variable received the lowest weight. In the Combined LDA Analysis, significant discrimination occurred in the second (1.5-2 hours) and third (2.5-3 hours) post treatment intervals only (binomial $p<.001$ ). This analysis used three of the variables included in the EEG analysis (posterior delta power during the eyes open state, the difference in frontal midline theta power between the low load and high load task, and the difference in alpha power be-

Table 1. Direction of Significant $(p<.05)$ Change in Spectral Power (vs. placebo) for Each EEG Feature in Each Treatment Condition

\begin{tabular}{|c|c|c|c|}
\hline & Caffeine & Alcohol & Diphenhydramine \\
\hline \multicolumn{4}{|c|}{ Resting EEG, Eyes Closed } \\
\hline Pz delta & - & $\Delta$ & $\boldsymbol{\Delta}$ \\
\hline Pz theta & - & $\Delta$ & - \\
\hline Oz alpha & - & - & $\boldsymbol{\nabla}$ \\
\hline \multicolumn{4}{|c|}{ Resting EEG, Eyes Open } \\
\hline Pz delta & - & $\Delta$ & $\boldsymbol{\Delta}$ \\
\hline Pz theta & - & $\Delta$ & - \\
\hline Oz alpha & - & - & - \\
\hline \multicolumn{4}{|c|}{ Task EEG, Low Load } \\
\hline aFz theta & - & $\Delta$ & - \\
\hline Pz alpha & $\boldsymbol{\nabla}$ & $\Delta$ & - \\
\hline \multicolumn{4}{|c|}{ Task EEG, High Load } \\
\hline $\mathrm{aFz}$ theta & - & $\Delta$ & $\boldsymbol{\nabla}$ \\
\hline Pz alpha & $\boldsymbol{\nabla}$ & $\Delta$ & - \\
\hline
\end{tabular}




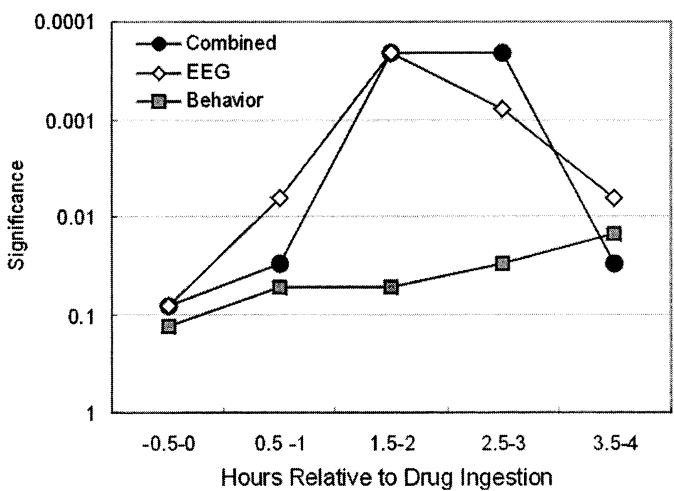

Figure 4. Binomial significance of the cross-validated classification outcomes of the linear discriminant functions distinguishing data obtained in the caffeine condition from that obtained in the placebo condition, using three types of indices. The Behavioral index used working memory task performance features, the EEG index used EEG features recorded during task performance or passive resting states. The Combined index used both performance and EEG features. It was not possible to discriminate caffeine from placebo using just behavioral measures. In contrast, the conditions could be discriminated in each of the four post-drug intervals using EEG measures, or in the first three post-drug intervals using combined EEG and behavioral measures.

tween the resting, eyes open state and performance of the high load task) in addition to a behavioral variable (reaction time variability in the high load task). Again the highest weight was given to the resting EEG variable, with the task-related frontal midline theta variable receiving the second highest weight, and the task-alpha variable receiving the lowest weight.

\section{Multivariate Detection of Treatment with Alcohol}

The Behavior LDA Analysis produced significant (binomial $p<.01)$ discrimination between the alcohol and placebo conditions in the second (1.5-2 hrs post drug) post-treatment interval only. This analysis used two behavioral features, reaction time variability in the low load level of the task, and a measure of the speed/accuracy tradeoff (reaction time divided by accuracy) in the high load level of the task. At the interval of significant discrimination, both variables were almost equally weighted in the discriminant equation. In contrast, the EEG LDA analysis showed significant discrimination (binomial $p<.001$ ) between alcohol and placebo conditions in all post-treatment intervals, with the peak difference occurring 2.5 to $3 \mathrm{hrs}$ post treatment. This analysis used two alpha features in the eyes closed resting state: one recorded over frontal areas and the other recorded over occipital areas. It also used alpha over frontal areas during performance of the low load task, and posterior theta power during performance of the high load task. The highest weight was given to the frontal alpha measures, with the resting alpha receiving the highest weight during the first two post drug intervals and the task alpha receiving the highest weight during the third and fourth post drug intervals. The remaining two measures received equivalent low weightings for all four post drug intervals. The Combined LDA used the same three alpha features as the EEG LDA analyses and also used a behavioral measure: average reaction time divided by reaction time variability in the high load task, although this feature received a very low weight in the equations. The results of the Combined LDA were very similar to those of the EEG LDA (Figure 5), and the three EEG measures received similar relative weightings as in the EEG LDA.

\section{Multivariate Detection of Treatment with Diphenhydramine}

All three sets of analyses produced significant discrimination between the diphenhydramine and placebo conditions (Figure 6). The Behavior Analysis used two variables (reaction time variability in the low load task and response accuracy in the high load task; the former received the highest weighting in the equations). Significant classification was only obtained in the second and third post-drug intervals, with peak discrimination occurring in the third post drug interval (binomial $p<$ .001). Discrimination returned towards chance levels in the final post-drug interval (3.5-4 hrs post drug). The EEG Analysis used two second-order task-related EEG

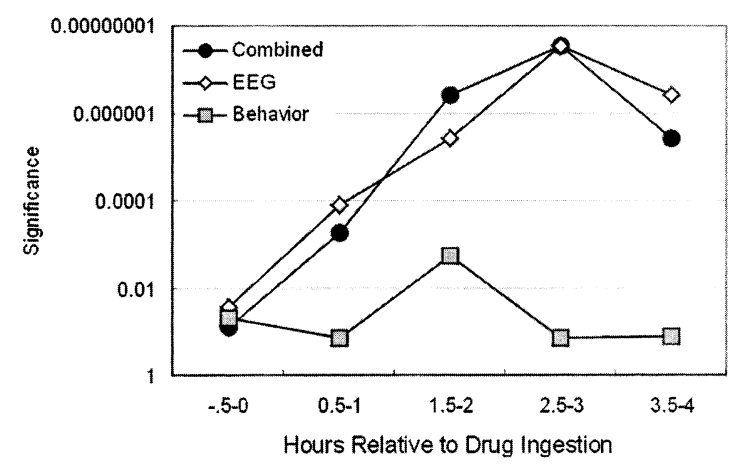

Figure 5. Binomial significance of the cross-validated classification outcomes of the linear discriminant functions distinguishing data obtained in the alcohol condition from that obtained in the placebo condition, using three types of indices. The Behavioral index used working memory task performance features, the EEG index used EEG features recorded during task performance or passive resting states. The Combined index used both performance and EEG features. Using behavioral features alone, the two conditions could be discriminated only in the second post treatment interval, occurring 1.5 to $2 \mathrm{hr}$ post drug ingestion. In contrast, the indices using EEG features showed significant discrimination between the two conditions in all post treatment intervals. 


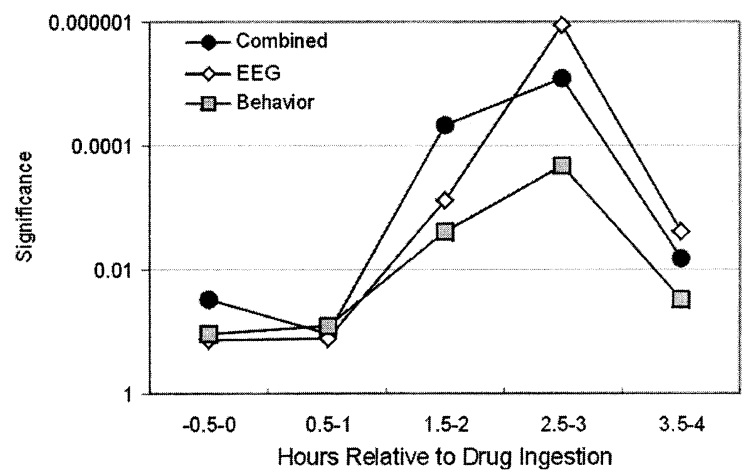

Figure 6. Binomial significance of the cross-validated classification outcomes of the linear discriminant functions distinguishing data obtained in the diphenhydramine condition from that obtained in the placebo condition, using three types of indices. The Behavioral index used working memory task performance features, the EEG index used EEG features recorded during task performance or passive resting states. The Combined index used both performance and EEG features. Although all three indices showed significant discrimination beginning in the interval 1.5 to $2 \mathrm{hr}$ post drug ingestion, the Combined index showed the highest level of discrimination at this point. All indices showed peak discrimination in the interval 2.5 to $3 \mathrm{hr}$ post drug. The EEG and Combined index showed significant discrimination in the final interval (3.5-4 hr post drug) but the Behavioral index did not.

variables and two second-order resting EEG variables. The task related variables included the difference in frontal midline theta power between the low load and high load task and the difference in alpha power between the resting, eyes open state and performance of the high load task. The resting EEG variables included the ratio of theta to alpha power over occipital channels in the eyes open state and the equivalent ratio in the eyes closed state. It revealed a similar pattern of discrimination as the Behavior Analysis, although with greater classification accuracy (binomial $p<.00001$ ) at the peak interval and with significant discrimination extending through the final post-drug interval (3.5-4 hrs post drug; binomial $p<.01$ ). The highest weightings were given to the resting EEG variables, followed by the frontal midline theta feature.

The Combined Analysis used one behavioral feature (reaction time variability in the low load task), two second order task-related EEG features (the difference in frontal midline theta power between the low load and high load task and the difference in alpha power between the resting, eyes open state and performance of the high load task) and one second-order resting EEG variable (the ratio of theta to alpha power over occipital channels in the eyes closed state). It produced better discrimination between diphenhydramine and placebo than did the other two analyses at the second post-drug interval (1.5-2 $\mathrm{hr}$ post drug; binomial $p<.0001)$. This analysis also showed peak discrimination during the third post drug interval $(2.5-3 \mathrm{Hr}$ post drug, binomial $p<.00001)$, and significant discrimination during the final interval (3.5-4 hr post drug; binomial $p<.01)$. Again the resting EEG feature received the highest weighting, with the other three features receiving equivalent moderate weights. As with the analysis of the caffeine data, this analysis also resulted in the selection of both EEG and behavioral variables, again suggesting that the two classes of inputs provided complementary rather than redundant information.

\section{Retest Reliability of Diphenhydramine Effects}

The eleven subjects who participated in the retest of the diphenhydramine treatment condition experienced similar levels of subjective drowsiness in the retest session as in the first diphenhydramine session. With respect to behavioral performance, subjects also showed similar increases in reaction time and decreases in accuracy in the retest session as in the original diphenhydramine session, with significant performance decrements in the second and third recording interval. EEG variables also showed similar effects between the two test sessions. That is, diphenhydramine was associated with an increase in the incidence of power in the delta and theta EEG bands during both resting conditions, attenuation of power in alpha band in the eyes-closed resting condition, and a relative reduction in power for the frontal midline theta rhythm in the high load task.

Two approaches were used to examine the reliability of the multivariate method for detecting the effects of diphenhydramine. In the first, we attempted to discriminate the data obtained from the original diphenhydramine session from that obtained in the second diphenhydramine session for the 11 subjects who participated in the retest condition. No variables could be found to discriminate between the two data sets, signifying that there were no systematic differences between the retest diphenhydramine data and the original data. In a second analysis, we tested the reliability of the multivariate method for discriminating drug data from placebo data. For this analysis, we first recomputed the Combined Analysis (described above) to discriminate the original diphenhydramine data from the placebo data using only the 11 subjects who participated in both the original and retest sessions. Since there were fewer subjects in this analysis than in the original analysis $(\mathrm{n}=$ 11 vs. $\mathrm{n}=16$ ), we restricted the discrimination function to a maximum of three variables. The recomputed index again included both EEG and behavioral variables, and it showed significant discrimination of drug from placebo data beginning at 1.5 hours post drug and lasting until the end of the session, with peak discrimination occurring at 2.5-3 hours post drug. The function 
obtained on the original data was then used to discriminate the retest data from the placebo data. Similar levels of discrimination were found for the retest data as for the original data (Figure 7), confirming that the effects of diphenhydramine on EEG and behavioral variables were very similar in the two sessions.

\section{DISCUSSION}

The objective of this study was to determine whether multivariate pattern classification methods applied to combinations of EEG measures and measures of overt task performance could be used to detect the acute CNS effects of common psychoactive substances (caffeine, alcohol, and the antihistamine diphenhydramine) and to characterize their pharmacodynamics over an extended test session. The pharmacological interventions produced changes in behavior and brain function consistent with past studies of their effects. For each treatment, multivariate detection functions could be derived that were sensitive and specific, and the cross-validation strategy indicated that such functions could generalize to data from new subjects. These findings are discussed below.

\section{Changes in Task Performance Following Treatment with Caffeine, Alcohol, or Diphenhydramine}

The observed effects of the pharmacological treatments on task performance are largely consistent with the findings of past studies. Caffeine increases arousal, reduces fatigue, and, in moderate doses, can speed responses and improve performance on attention tasks (Jacobson and Edgley 1987; Jarvis 1993; Lorist et al. 1996). In the current study, subjects performed the tasks slightly

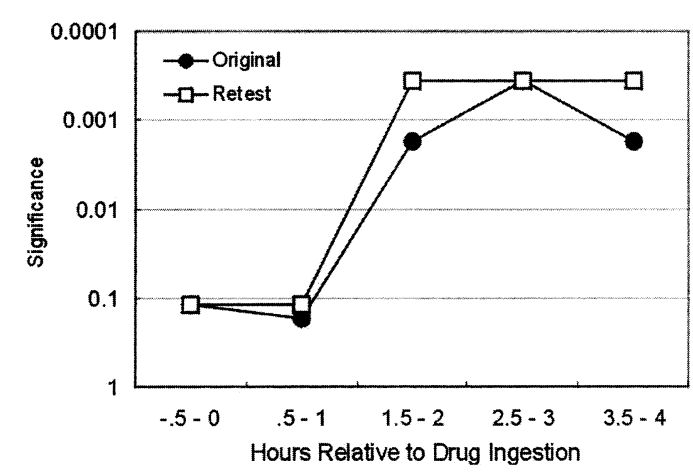

Figure 7. Binomial significance of the linear discriminant functions discriminating placebo data from the first (original) and second (retest) diphenhydramine conditions, using a Combined Index composed of behavioral and electrophysiological variables. The LDA was performed on the original data for the 11 subjects participating in the retest condition, and then validated by application to the retest data. faster and more accurately following caffeine ingestion, but this trend was not statistically significant. This lack of a performance enhancing effect of caffeine is not surprising given the test conditions employed here. First, the subjects had been highly practiced on the tasks on several occasions prior to the point at which the critical data were collected; because of this extensive practice their accuracy and response speed had likely reached asymptotic levels. In this respect these data are equivalent to the negative results described by Lorist and Snel (1997), who also failed to find significant performance enhancing effects of caffeine in highly practiced subjects. Second, performance improvements with caffeine ingestion are typically larger in fatigued subjects (Lorist et al. 1994a, 1994b). In the current study, subjects were well rested at the beginning of the test sessions, and subjective ratings suggest that they did not experience any significant increase in fatigue over the course of the test session in the placebo or caffeine conditions.

Alcohol is a central nervous system depressant. In past studies of the cognitive effects of alcohol it has been observed to slow psychomotor responses and to decrease accuracy in simple vigilance and sustained attention tasks (Koelega 1995; Rohrbaugh et al. 1988), and to modify response biases in immediate memory and continuous performance tasks (Dougherty et al., 2000). Such effects were most reliably observed at dose levels approximately $20 \%$ higher than those used in the current study. At the dose level employed in the current study, alcohol did not significantly reduce accuracy but it did have complex effects on response speed, slowing responses to stimuli in the low load task and speeding them in the high load task. Relatively subtle effects of low doses of alcohol on performance were also found in a prior study that utilized the same tasks employed here (Gevins and Smith 1999).

The antihistamine diphenhydramine has been noted to produce subjective sedation and to impair cognitive function, particularly in tasks that require sustained attention and speeded visual-motor responses (Fine et al. 1994a; Gengo et al. 1989; Gengo et al. 1990; Kay et al. 1997; Moskowitz and Burns 1988; Rice and Snyder 1993; Witek et al. 1995). In the current study we found that responses were significantly slower and less accurate following ingestion of diphenhydramine relative to the other test conditions. Similar effects have been observed in prior studies that have examined performance following diphenhydramine administration in subject populations and under dosing ranges and procedural conditions approximating those in the current study (Fine et al. 1994b; Oken et al. 1995; Rice and Snyder 1993; Tharion et al. 1994). Of particular interest is the observation that the behavioral impairment produced by $50 \mathrm{mg}$ of diphenhydramine was substantially greater than the behavioral change observed following a dose of alcohol adequate to raise blood alcohol to the 
level of legal intoxication in many states. This finding is consistent with another recent study that reported that simulated driving performance was worse following treatment with diphenhydramine than following a legally intoxicating dose of alcohol (Weiler et al., 2000).

\section{Changes in Neurophysiological Activity Following Treatment with Caffeine, Alcohol, or Diphenhydramine}

The particular measures of brain electrical activity made in this study were selected on the basis of past studies that have shown them to be sensitive to variations in alertness or attentional effort (Oken and Salinsky 1992). For example, past studies have shown that in the EEG recorded under passive resting conditions, drowsiness is associated with an increase in spectral power in the delta band $(<4 \mathrm{~Hz})$ and the lower portion $(4-6 \mathrm{~Hz})$ of the theta band, and with attenuation of alpha band $(8-12 \mathrm{~Hz})$ signals under eyes closed states (Davis et al. 1937; Gevins et al. 1977; Makeig and Jung 1995; Matousek and Petersen 1983; Oken and Salinsky 1992). Past studies with the tasks employed here have also identified signals that vary systematically with changes in task difficulty and hence the degree of attentional effort demanded for accurate task performance (Gevins and Smith 1999; Gevins and Smith, 2000; Gevins et al. 1996, 1997, 1998; McEvoy et al. 1998; McEvoy et al., 2000). In particular, such studies have demonstrated that the frontal midline theta $(5-7 \mathrm{~Hz})$ rhythm tends to be larger in the high load task condition, whereas the lower portion $(8-10 \mathrm{~Hz})$ of the alpha band tends to be attenuated with increased task difficulty.

Because the neurophysiological measures described above are sensitive to variations in alertness or attentional effort, it was anticipated that they might also be affected by the pharmacological treatments that were introduced. The results confirmed this expectation. Caffeine, alcohol, and diphenhydramine each produced a distinct pattern of changes in these variables. Consistent with the data on subjective alertness and with the overt performance results, the neurophysiological measures recorded following treatment with caffeine differed relatively little from those recorded in the placebo condition. In the eyes-closed resting data, caffeine tended to be associated with a decrease in EEG activity in the delta and theta bands, and an increase in EEG activity in the alpha band. While these findings are suggestive of increased alertness, the changes were not statistically reliable across the group of subjects. The only significant change produced by caffeine was a reduction in alpha band activity during task performance, a result suggesting that subjects were somewhat more attentive to task performance than they were under placebo conditions.

In contrast, alcohol produced large significant effects on both the resting and task related EEG. These effects were disproportionate to the relatively subtle behavioral changes it produced. During both resting conditions the largest alcohol-related changes were an increase in spectral power in the delta and theta bands. While such a change is consistent with a relative decrease in arousal, such an inference must be made with caution given the absence of neurophysiological signs of drowsiness characteristic of passive resting conditions. During both task performance conditions alcohol was associated with an increase in spectral power for the frontal midline theta rhythm and in the lower portion of the alpha band. Since these changes occurred independently of the task demands placed on the subject, they are unlikely to reflect specific changes in the way that attentional resources were allocated to the tasks. Rather, the general increase in rhythmic EEG activity following alcohol ingestion observed here and in other studies (Cohen et al. 1993; Davis et al. 1941; Gevins and Smith 1999; Lukas et al. 1986) suggests that acute treatment with alcohol changes the intrinsic oscillatory properties of cortical neurons.

Past studies have indicated that diphenhydramine produces changes in EEG signals that are similar to those associated with increased drowsiness (Oken et al. 1995). The data from the current experiment are consistent with this view. In particular, during resting conditions treatment with diphenhydramine produced an increase in power in the delta band, a decrease in alpha band power during eyes-closed conditions, and an increase in the incidence of slow eye movement activity. These changes in neurophysiological indicators of drowsiness are consistent with the subjective reports of increased drowsiness following diphenhydramine ingestion, and with the impaired task performance. In contrast to the effects of alcohol, diphenhydramine had relatively subtle and specific effects on the task-related EEG. In particular, diphenhydramine attenuated the increased power for the frontal midline theta rhythm that is otherwise observed in the high load task relative to the low load task. To the extent to which the typical task load-related increase in this signal reflects effortful attention, this task-specific increase suggests that following diphenhydramine administration subjects were unable or unwilling to expend additional mental effort to confront the increase in task demands.

\section{Multivariate Detection of Drug Effects}

The LDA analyses performed in the current study established that the types of pharmacologically-induced behavioral and EEG changes described above are robust enough to be detected in most individual subjects. Moreover, for each treatment intervention the sensitivity of the classification function was greatly improved by the inclusion of EEG measures. For caffeine, it was not possible to derive a function capable of discriminat- 
ing treatment and placebo data at above chance levels during any time period when only behavioral measures were used. In contrast, when EEG and behavioral measures were both included in the LDA, highly significant detection of the caffeine treatment was obtained. Similarly, an LDA restricted to behavioral measures was only able to detect treatment with alcohol at a weakly significant level in one post-treatment interval; with the addition of EEG variables highly significant classification was achieved in all post-treatment intervals. Even in the case of diphenhydramine, where treatment was found to produce significant response slowing and decreased accuracy relative to the placebo condition, the addition of EEG variables was found to dramatically improve the sensitivity of LDA based classification functions. Together these results provide strong evidence that there is substantial value added by the inclusion of physiological measures of brain function in efforts to sensitively characterize the pharmacodynamics of psychoactive substances.

The results also suggest that there is value to recording the EEG during resting states as well as during active task performance conditions. When considering the treatment effects on univariate neurophysiological parameters, each treatment was found to produce a distinct pattern of changes across the various test conditions. For example, for caffeine, EEG parameters most reliably differed from placebo during performance of both low load and high load WM tasks, but not during resting states. For alcohol, EEG parameters reliably differed from placebo during both resting states and performance of both WM task versions. For diphenhydramine, EEG parameters reliably differed from placebo during resting states and selectively during performance of the high load WM task. These different patterns of results indicate that the neurophysiological changes that accompany a particular psychoactive treatment are dependent upon the functional demands placed on the individual being tested. Furthermore, each stepwise LDA analysis performed using neurophysiological measures as input variables yielded classification functions that included combinations of resting and task-related data. This pattern of results implies that measures of changes in EEG variables across levels of functional demand provide particularly sensitive indices of the way in which brain function is affected by pharmacological interventions.

Finally, even though examples of a particular subject's behavior and EEG data were not used in the development of the functions used to classify their data, a high degree of classification accuracy was nonetheless achieved. The successful leave-out-one-subject crossvalidation strategy indicated that there was a good deal of commonality across individuals in the characteristic effects of the treatments, and that the classification functions did not just reflect serendipitous fits to the particular training datasets from which they were derived. Furthermore, the successful cross-validation of a combined function derived from the first test day with diphenhydramine accomplished by applying it to the data from the diphenhydramine retest session illustrates the high degree of reliability in neurocognitive responses to the pharmacological treatment.

\section{CONCLUSIONS}

The results from this study lend themselves to several conclusions. First, whereas behavioral measures are sometimes adequate for detecting the effect of a pharmacological treatment on cognitive function, it is possible to detect such effects with much greater sensitivity with the addition of EEG measures of brain activity. Second, different pharmacological interventions appear to elicit different patterns of EEG changes depending upon whether or not an individual is actively engaged in task performance. As a result, there is also a benefit to classifier performance derived from including samples of both resting and task related EEG data. Third, the behavioral and EEG effects produced by pharmacological interventions appear to be fairly homogenous across individuals and fairly stable within individuals across multiple test sessions. Because of this stability it is possible that standardized multivariate detectors of EEG and behavioral changes could be used to characterize the cognitive effects of particular pharmacological interventions across time, experiments, and laboratories. Such results provide compelling evidence to suggest that physiological indices add substantial value when assessing the neurocognitive effects of pharmacological interventions. They also suggest that the multivariate procedures used here could be developed into a sensitive test of the psychoactive properties of new drugs, or of existing drugs that have effects that are less well understood than those observed for caffeine, alcohol, and diphenhydramine.

\section{ACKNOWLEDGMENTS}

We thank Jennifer Barber, Halle Brown, Gail Chang, Arati Karnik, Caroline Prioleau, and Georgia Rush for assistance with data collection and analysis. The National Aeronautics and Space Administration, the National Institute of Mental Health, and the Air Force Office of Scientific Research sponsored this research.

\section{REFERENCES}

Åkerstedt T, Gillberg M (1990): Subjective and objective sleepiness in the active individual. Int J Neursci 52:29-37

Broughton R (1982): Performance and evoked potential mea- 
sures of various states of daytime sleepiness. Sleep 5:135-146

Bruce M, Scott N, Lader M, Marks V (1986): The psychopharmacological and electrophysiological effects of single doses of caffeine in healthy human subjects. Br J Clin Pharmacol 22:81-87

Cohen HL, Porjesz B, Begleiter H (1993): The effects of ethanol on EEG activity in males at risk for alcoholism. Electroenceph Clin Neurophysiol 86:368-376

Davis H, Davis PA, Loomis AL, Harvey EN, Hobart G (1937): Human brain potentials during the onset of sleep. J Neurophysiol 1:24-37

Davis PA, Gibbs FA, Davis H, Jetter WW, Trowbridge LS (1941): The effects of alcohol upon the electroencephalogram (brain waves). Q J Stud Alc 1:626-637

Dougherty DM, Marsh DM, Moeller FG, Chokshi RV, Rosen VC (2000): Effects of moderate and high doses of alcohol on attention, impulsivity, discriminability, and response bias in immediate and delayed recall performance. Alcohol Clin Exp Res 24:1702-1711

Du W, Leong H, Gevins AS (1994): Ocular artifact reduction by adaptive filtering. Paper presented at the Seventh IEEE SP Workshop on Statistical Signal and Array Processing, June 26-29, Quebec City, Quebec, Canada

Efron B (1982): The Jackknife, the Bootstrap and other Resampling Plans. Philadelphia, Society for Industrial and Applied Mathematic

Fine BJ, Kobrick JL, Lieberman HR, Marlowe B, Riley RH, Tharion WJ (1994a): Effects of caffeine or diphenhydramine on visual vigilance. Psychopharmacology (Berl) 114:233-238

Fine BJ, Kobrick JL, Lieberman HR, Marlowe B, Riley RH, Tharion WJ (1994b): Effects of caffeine or diphenhydramine on visual vigilance. Psychopharmacology (Berl) 114:233-238

Fireman P (1997): Treatment of allergic rhinitis: effect on occupation productivity and work force costs. Allergy Asthma Proc 18:63-67

Gale A, Davies I, Smallbone A (1978): Changes in the EEG as the subject learns to recall. Biol Psychol 6:169-179

Galin D, Johnstone J, Herron J (1978): Effects of task difficulty on EEG measures of cerebral engagement. Neuropsychol 16:461-472

Gengo F, Gabos C, Miller JK (1989): The pharmacodynamics of diphenhydramine-induced drowsiness and changes in mental performance. Clin Pharmacol Ther 45:15-21

Gengo FM, Gabos C, Mechtler L (1990): Quantitative effects of cetirizine and diphenhydramine on mental performance measured using an automobile driving simulator. Ann Allergy 64:520-526

Gevins A, Smith ME (1999): Detecting transient cognitive impairment with EEG pattern recognition methods. Aviat Space Env Med 70:1018-1024

Gevins A, Smith ME (2000): Neurophysiological measures of working memory and individual differences in cognitive ability and cognitive style. Cereb Cortex 10:829-839

Gevins A, Smith ME, Leong H, McEvoy L, Whitfield S, Du R, Rush G (1998): Monitoring working memory load during computer-based tasks with EEG pattern recognition methods. Hum Fact 40:79-91
Gevins A, Smith ME, McEvoy L, Yu D (1997): High resolution EEG mapping of cortical activation related to working memory: effects of task difficulty, type of processing, and practice. Cereb Cortex 7:374-385

Gevins AS, Smith ME, Le J, Leong H, Bennett J, Martin N, McEvoy L, Du R, Whitfield S (1996): High resolution evoked potential imaging of the cortical dynamics of human working memory. Electroenceph Clin Neurophysiol 98:327-348

Gevins AS, Stone RK, Ragsdale SD (1988): Differentiating the effects of three benzodiazepines on non-REM sleep EEG spectra. Neuropsychobiol 19:108-115

Gevins AS, Zeitlin GM, Ancoli S, Yeager CL (1977): Computer rejection of EEG artifact. II: Contamination by drowsiness. Electroenceph Clin Neurophysiol 42:31-42

Haring C, Neudorfer C, Schwitzer J, Hummer M, Saria A, Hinterhube H, Fleischhacker WW (1994): EEG alterations in patients treated with clozapine in relation to plasma levels. Psychopharmacology (Berl) 114:97-110

Hermann WM (1982): Development and critical evaluation of an objective procedure for the electroencephalographic classification of psychotropic drugs. In Hermann WM (ed), EEG in Drug Research, New York, Gustav Fisher, pp 249-351

Hoddes E, Zarcone V, Smythe H, Phillips R, Dement WC (1973): Quantification of sleepiness: a new approach. Psychophysiol 10:431-436

Inouye T, Ishihara T, Shinosaki K, Toi S, Satoshi U (1988): EEG characteristics of frontal midline theta activity. In Giannitrapani M (ed), The EEG of Mental Activities, Basel, Karger, pp 136-148

Jacobson BH, Edgley BM (1987): Effects of caffeine on simple reaction time and movement time. Avia Space Environment Med 58:1153-1156

Jarvis MJ (1993): Does caffeine intake enhance absolute levels of cognitive performance? Psychopharmacology 110: $42-52$

Kay GG, Berman B, Mockoviak SH, Morris CE, Reeves D, Starbuck V, Sukenik E, Harris AG (1997): Initial and steady-state effects of diphenhydramine and loratadine on sedation, cognition, mood, and psychomotor performance [see comments]. Arch Intern Med 157:2350-2356

Koelega HS (1995): Alcohol and vigilance performance: a review. Psychopharmacology (Berl) 118:233-249

Lorist MM, Snel J (1997): Caffeine effects on perceptual and motor processes. Electroenceph Clin Neurophysiol 102:401-413

Lorist MM, Snel J, Kok A (1994a): Influence of caffeine on information stages in well rested and fatigued subjects. Psychopharmacology (Berl) 113:411-421

Lorist MM, Snel J, Kok A, Mulder G (1994b): Influence of caffeine on selective attention in well rested and fatigued subjects. Psychophysiol 31:525-534

Lorist MM, Snel J, Kok A, Mulder G (1996): Acute effects of caffeine on selective attention and visual search processes. Psychophysiol 33:354-361

Lukas SE, Mendleson JH, Benedilt RA, Jones B (1986): EEG alpha activity increases during transient episodes of ethanol-induced euphoria. Pharmacol Biochem Behav 25:889-895 
Makeig S, Jung TP (1995): Changes in alertness are a principal component of variance in the EEG spectrum. Neuroreport 7:213-216

Matousek M, Petersen IA (1983): A method for assessing alertness fluctuations in vigilance and the EEG spectrum. Electroenceph Clin Neurophysiol 55:108-113

McEvoy LK, Smith ME, Gevins A (1998): Dynamic cortical networks of verbal and spatial working memory: effects of memory load and task practice. Cereb Cortex 8:563-574

McEvoy LK, Smith ME, Gevins A (2000): Test-retest reliability of cognitive EEG. Clin Neurophysiol 111:457-463

Miyata Y, Tanaka Y, Hono T (1990): Long term observation on Fm-theta during mental effort. Neurosci 16:145-148

Moskowitz H, Burns M (1988): Effects of terfenadine, diphenhydramine, and placebo on skills performance. Cutis 42:14-18

Oken BS, Kishiyama SS, Salinsky MC (1995): Pharmacologically induced changes in arousal: effects on behavioral and electrophysiologic measures of alertness and attention. Electroenceph Clin Neurophysiol 95:359-371

Oken BS, Salinsky M (1992): Alertness and attention: basic science and electrophysiologic correlates. J Clin Neurophysiol 9:480-494

Ramaekers J (1998): Behavioral toxicity of medicinal drugs. Practical consequences, incidence, management and avoidance. Drug Saf 18:189-208

Rice VJ, Snyder HL (1993): The effects of Benadryl and Hismanal on psychomotor performance and perceived performance. Aviat Space Environ Med 64:726-734

Rohrbaugh JW, Stapleton JM, Parasuraman R, Frowein HW, Adinoff B, Varner JL, Zubovic EA, Lane EA, Eckardt MJ, Linnoila M (1988): Alcohol intoxication reduces visual sustained attention. Psychopharmacology (Berl) 96:442-446

Saletu B, Grunberger J, Linzmayer L, Semlitsch HV, Anderer P, Chwatal K (1994): Pharmacokinetic and dynamic studies with a new anxiolytic, suriclone, utlizing EEG mapping and psychometry. Br J Clin Pharmacol 37:145-156

Salinsky MC, Oken BS, Morehead L (1991): Test-retest reliability in EEG frequency analysis. Electroenceph Clin Neurophysiol 79:383-392

Schulz H, Jobert M, Coppola R, Herrmann WM, Pantev M (1996): The use of diurnal vigilance changes in the EEG to verify vigilance-enhancing effects of memantine in a clinical pharmacological study. Neuropsychobiol 33:32-40
Semlitsch HV, Anderer P, Saletu B, Binder GA, Decker KA (1995): Cognitive psychophysiology in nootropic drug research: effects of Ginkgo biloba on event-related potentials (P300) in age-associated memory impairment. Pharmacopsychiatry 28:134-142

Smith ME, McEvoy LK, Gevins A (1999): Neurophysiological indices of strategy development and skill acquisition. Cogn Brain Res 7:389-404

Sumner DD (1998): Benzodiazepine-induced persisting amnestic disorder: are older adults at risk? Arch Psychiatr Nurs 12:119-125

Swets JA (1964): Signal Detection and Recognition by Human Observers. New York, Wiley

Tharion WJ, McMenemy DJ, Rauch TM (1994): Antihistamine effects on the central nervous system, cognitive performance, and subjective states. Neuropsychobiol 29:97-104

Torsvall L, Åkerstedt T (1988): Extreme sleepiness: quantification of EOG and spectral EEG parameters. Int J Neursci 38:435-441

Valentine AD, Meyers CA, Kling MA, Richelson E, Hauser P (1998): Mood and cognitive side effects of interferonatherapy. Semin Oncol 25(Suppl 1):39-47

van Dam FS, Schagen SB, Muller MJ, Boogerd W, van den Wall E, Droogleever Fortuyn ME, Rodenhuis S (1998): Impairment of cognitive function in women receiving adjuvant treatment for high-risk breast cancer: high does-versus standard-dose chemotherapy. J Natl Cancer Inst 90:210-218

Vermeulen J, Aldenkamp AP (1995): Cognitive side-effects of chronic antiepileptic drug treatment: a review of 25 years of research. Epilepsy Research 22:65-95

Veselis RA, Reinsel R, Wronski M (1993): Analytic methods to differentiate similar electroencephalographic spectra: neural networkj and discriminant analysis. J Clin Monit 9:257-267

Weiler JM, Bloomfield JR, Woodworth GG, Grant AR, Layton TA, Brown TL, McKenzie DR, Baker TW, Watson GS (2000): Effects of fexofenadine, diphenhydramine, and alcohol on driving performance. A randomized, placebo-controlled trial in the Iowa driving simulator. Ann Intern Med 132:354-363

Witek TJ Jr, Canestrari DA, Miller RD, Yang JY, Riker DK (1995): Characterization of daytime sleepiness and psychomotor performance following $\mathrm{H} 1$ receptor antagonists. Ann Allergy Asthma Immunol 74:419-426 